\title{
Correction to: The Effect of Maternal Self-Regulated Motivation on Breastfeeding Continuation
}

\author{
Christine Y. K. Lau ${ }^{1,5}$ (1) - Daniel. Y. T. Fong ${ }^{1}$ - Vincci. H. S. Chan ${ }^{1,6} \cdot$ Kris. Y. W. Lok ${ }^{1} \cdot$ Judy. W. Y. Ng ${ }^{2,7} \cdot$ Chu Sing $^{3,8}$. \\ Marie Tarrant ${ }^{4}$
}

Published online: 4 January 2022

○) Springer Science+Business Media, LLC, part of Springer Nature 2021

\section{Correction to: Maternal and Child Health Journal https://doi.org/10.1007/s10995-021-03274-5}

Unfortunately the article was published with a spelling error in the co-author name "Marrie Tarrant". The correct coauthor name should be "Marie Tarrant".

Publisher's Note Springer Nature remains neutral with regard to jurisdictional claims in published maps and institutional affiliations.

The original article can be found online at https://doi.org/10.1007/ s10995-021-03274-5.

Christine Y. K. Lau

cyklau@connect.hku.hk

1 School of Nursing, Li Ka Shing Faculty of Medicine, University of Hong Kong, Hong Kong SAR, People's Republic of China

2 Department of Obstetrics and Gynecology, Princess Margaret Hospital, Hong Kong SAR, People's Republic of China

3 Department of Obstetrics and Gynecology, Kwong Wah Hospital, Hong Kong SAR, People's Republic of China

4 School of Nursing, Okanagan Campus, University of British Columbia, Kelowna, Canada
5 Present Address: Jockey Club School of Public Health and Primary Care, Faculty of Medicine, The Chinese University of Hong Kong, Hong Kong SAR, People's Republic of China

6 Present Address: Faculty of Social Science, The Chinese University of Hong Kong, Hong Kong SAR, People's Republic of China

7 Present Address: Registered Midwife, Hong Kong SAR, People's Republic of China

8 Present Address: Maternity, Matilda International Hospital, Hong Kong SAR, People's Republic of China 